\title{
Automated Cryo-tomography and Single Particle Analysis with a New Type of Phase Plate
}

\author{
Radostin Danev ${ }^{1}$, Bart Buijsse ${ }^{2}$, Yoshiyuki Fukuda ${ }^{1}$, Maryam Khoshouei ${ }^{1}$, Juergen Plitzko ${ }^{1}$, Wolfgang \\ Baumeister $^{1}$
}

1. Max Planck Institute of Biochemistry, Martinsried, Germany.

2. FEI, Eindhoven, The Netherlands.

Recent years have shown an increased interest in the development and use of phase plates in cryo-EM. The oldest and the most productive type of phase plate is the carbon film Zernike phase plate [1]. It has been successfully used in cryo-tomography [2] and single particle analysis applications [3]. Despite its good performance the Zernike phase plate has a few pitfalls. One major practical hindrance is its short lifetime [4]. Typically within 10 days after being installed into the microscope its performance deteriorates to the point where it has to be exchanged. Another disadvantage of the Zernike phase plate is that it produces fringes around high-contrast features in the image, such as lipid membranes, support film edges etc [5]. Despite its shortcomings the Zernike phase plate has been the main motivation and experience generator in the last years.

We are currently working in collaboration with FEI on the development and testing of a new type of phase plate. It addresses both shortcomings of the Zernike phase plate discussed above. Our tests indicate that the new phase plate lasts for more than six months inside the microscope. This is a big advantage in terms of servicing and up time of the microscope. The other big advantage of the new phase plate is that it produces fringe-free images which resemble in appearance light microscope phase contrast images. The new phase plate is being developed as a part of a product package which will include new hardware - phase plate holder $\&$ phase plate, and new software for alignment, calibration and ease of use.

The new phase plate was tested with the two main 3D cryo-EM techniques - cryo-tomography and single particle analysis. Comparing the results with what has been published shows that it performs significantly better than the thin film Zernike phase plate. The improvements are mainly in image appearance and resolution.

For cryo-tomography the new phase plate was tested with two automated acquisition software packages - FEI Tomography and SerialEM. Both packages work well and are able to automatically acquire tilt series with the phase plate. There are only a couple of additional steps that are required for setting up the phase plate before starting the tilt series acquisition. The reconstruction process for the phase plate tomograms is almost identical to that for conventional defocus phase contrast tomograms and can be performed with any existing reconstruction software package (such as IMOD). Because of the strong low frequency components in the phase plate images a simple weighted back-projection reconstruction was in most cases sufficient to produce sharp, high-contrast tomograms. No further processing, such as de-noising, was necessary. In a few thick specimen cases a SIRT reconstruction produced better looking tomograms and again no de-noising or other post-processing was necessary. Overall the new phase plate works very well for cryo-tomography and users with cryo-tomography experience require only a short training on how to use it. 
For automated single particle image acquisition the new phase plate works well with the FEI EPU software. Similar to its use in tomography only a couple of additional steps are needed to set up the phase plate before starting the automated acquisition. Theoretically, for single particle analysis working in-focus and without CTF-correction should be the optimal application scenario for the phase plate. However our experience so far is that due to practical limitations to the focusing accuracy one has to still fit and demodulate the CTF in order to achieve the best resolution. Automatic fitting of the CTF of images taken with the phase plate is not supported by the built-in routines in the current versions of the popular single particle reconstruction packages. So we developed a software tool for fitting and signflipping of phase plate images. Each micrograph was sign-flip CTF-corrected before particle picking and the following reconstruction steps were carried out without any further CTF fitting or correction. The initial results (Tecnai F20, $200 \mathrm{kV}$ ) showed a resolution of $\sim 8 \mathrm{~A}$ and we expect that the ultimate resolution will be somewhat lower than what can be achieved with the conventional method. However, the extra contrast at low spatial frequencies can be valuable for the study of particles that are challenging to solve by the conventional defocus method, such as small, low-symmetry, heterogeneous or other samples.

The new phase plate was tested on two microscopes - a $200 \mathrm{kV}$ FEI Tecnai F20 and a $300 \mathrm{kV}$ FEI Titan Krios. The phase plate performance in terms of lifetime and image quality was equally good on both microscopes. The presence of a phase plate holder at the objective aperture position did not cause any side effects or performance degradation to the conventional operation of the microscopes.

[1] K Nagayama, J. Electron Microsc. 60(S1) (2011), p. S43.

[2] W Dai et al, Nature (2013), doi:10.1038/nature12604.

[3] R Danev and K Nagayama, J. Struct. Biol. 161 (2008), p. 211.

[4] R Danev, R Glaeser and K Nagayama, Ultramicroscopy 109 (2009), p. 312.

[5] R Danev and K Nagayama, Ultramicroscopy 111 (2011), p. 1305.
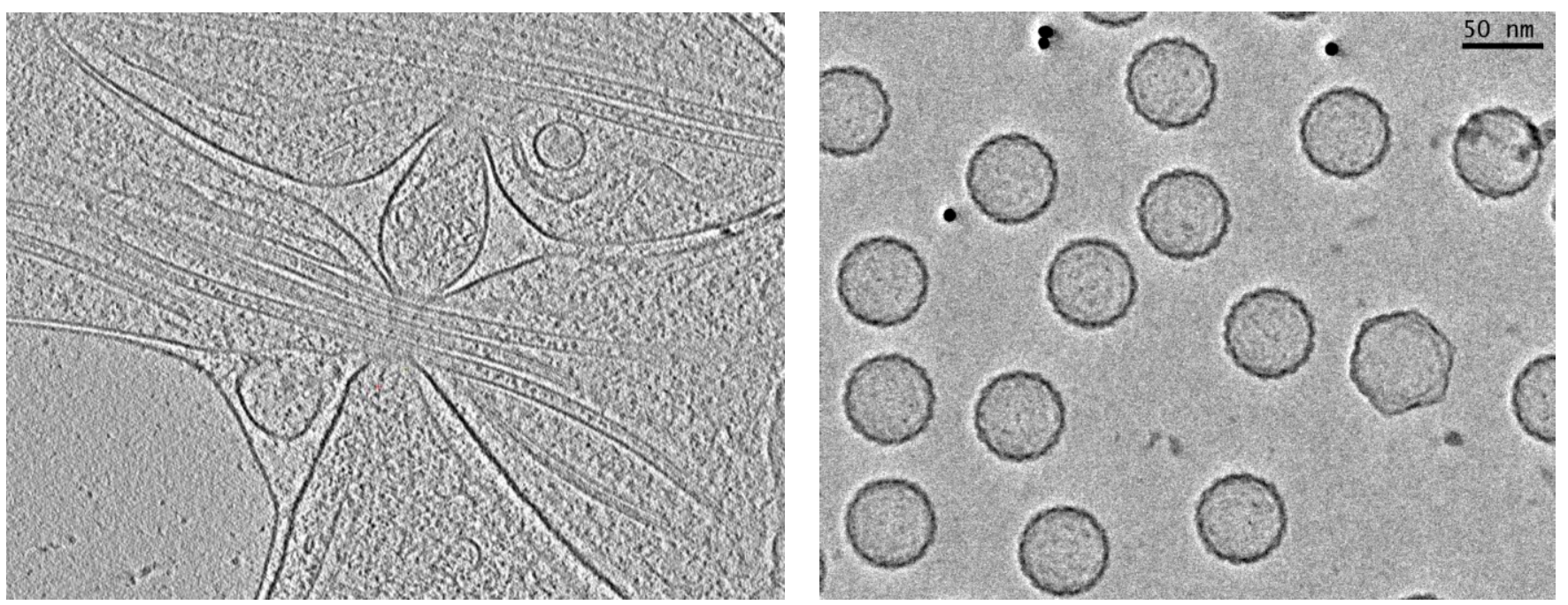

Figure 1. (left) A slice through a phase plate tomogram of a plunge-frozen primary neuron culture on a grid. (right) A single particle image of D3 virus capsids. Scale bars: $50 \mathrm{~nm}$. 